\title{
Thumb Involvement in Raynaud's Phenomenon as an Indicator of Underlying Connective Tissue Disease
}

\author{
BATSI CHIKURA, TONIA MOORE, JOANNE MANNING, ANDY VAIL, and ARIANE L. HERRICK
}

\begin{abstract}
Objective. To conduct a retrospective study to assess whether the degree of thumb involvement differs between primary Raynaud's phenomenon (PRP) and secondary Raynaud's phenomenon (SRP). Methods. Thermography images from all patients attending Salford Royal Hospital and referred for thermography for assessment of RP between 2004 and 2006 were retrospectively reviewed. A distal dorsal difference (DDD) of $-1^{\circ} \mathrm{C}$ or less between the fingertips and dorsum of the hand (fingers cooler) at $23^{\circ} \mathrm{C}$ was considered clinically relevant. The worse score (the lower score, i.e., the more negative value) from each pair of digits was considered for analysis.

Results. One hundred seventy patients fulfilled the study criteria. DDD at $23^{\circ} \mathrm{C}$ for the thumbs were significantly higher (digital tips warmer) compared with other digits $(\mathrm{p}<0.001)$ in both PRP and SRP. All digits were significantly warmer in PRP compared to SRP with the exception of the thumbs. The proportion of patients with clinically relevant involvement of thumbs was significantly higher in SRP compared to PRP ( $p=0.003$ ) and this difference was more pronounced in the thumbs compared with other digits.

Conclusion. Although the median temperature gradient along the thumb was not significantly different between SRP and PRP, the thumb is more likely to be involved in SRP than in PRP. Thumb involvement is one of a number of clinical indicators that should alert the clinician to the possibility of an underlying connective tissue disease/disorder. (First Release March 1 2010; J Rheumatol 2010;37:783-6; doi:10.3899/jrheum.091117)
\end{abstract}

\author{
Key Indexing Terms: \\ RAYNAUD'S PHENOMENON \\ SYSTEMIC SCLEROSIS
}

\section{THUMB SPARING THERMOGRAPHY \\ CONNECTIVE TISSUE DISEASES}

Raynaud's phenomenon (RP) is classically characterized by triphasic color changes in response to cold or emotional stress. RP can be divided into primary (PRP) and secondary $\mathrm{RP}$ (SRP) depending on the absence or presence of an underlying disorder, e.g., a connective tissue disease (CTD) such as systemic sclerosis $(\mathrm{SSc})^{1}$. Complete reversibility of episodic digital ischemia is an important characteristic of PRP and is a distinguishing feature from SRP in which irreversible tissue damage can occur.

In a prospective study ${ }^{2}$ we demonstrated that the thumb is spared in both PRP and SRP, as evidenced by patients' self-reported symptoms (subjective assessment) and thermography (objective assessment). On thermography, the thumb distal-dorsal difference (DDD) scores (measuring the

From the Department of Rheumatology, Lincoln County Hospital, Lincoln; Department of Rheumatic Diseases, Salford Royal NHS Foundation Trust, Salford; and the Health Methodology Research Group, University of Manchester, Manchester Academic Health Sciences Centre, Salford Royal Hospital, Salford, UK.

B. Chikura, MRCP UK, Consultant Rheumatologist; T. Moore, BSC, Senior Vascular Technician; J. Manning, HNC, Senior Vascular Technician; A. Vail, MSc, Senior Lecturer, Biostatistics; A.L. Herrick, MD, FRCP, Consultant Rheumatologist, Reader in Rheumatology.

Address correspondence to Dr. B. Chikura, Department of Rheumatology, Lincoln County Hospital, Greetwell Road, Lincoln, LN2 5QY, UK.

E-mail: batsi@doctors.org.uk

Accepted for publication November 30, 2009. temperature difference between the digital tip and the dorsum of the hand at $23^{\circ} \mathrm{C}$ ) were better, i.e., higher in PRP compared with SRP; however, there was no evidence that the degree of thumb-sparing was different between the PRP and SRP groups. We concluded that the sample size lacked enough power to answer this question. It is important to know whether the thumb is more involved in SRP compared to PRP; if so, this could point to an underlying CTD and this could help rheumatologists categorize patients. In this retrospective study we identified a large sample of patients with $\mathrm{RP}$ to determine whether the degree of thumb involvement differs between PRP and SRP.

\section{MATERIALS AND METHODS}

Patients. Patients were identified retrospectively on the basis that (1) they had undergone thermography at Salford Royal Hospital between 2004 and 2006; and (2) they satisfied our criteria for RP (at least one color change; white, blue, or red in response to cold as documented by the attending physician). Case notes were reviewed (electronic and/or paper) and patients were subdivided into those with PRP and with SRP. Patients with PRP satisfied the LeRoy and Medsger criteria ${ }^{1}$. Patients with SRP had either an identifiable underlying disorder (Table 1) or undifferentiated connective tissue disease (UCTD). All patients with UCTD were positive for antinuclear antibodies (ANA; positivity defined by $\operatorname{IgG}$ titer $\geq 1 / 100$ ). Some had positive antibodies to extractable nuclear antigens and some had abnormalities on nailfold microscopy.

The study was approved by the Warrington, Wigan and Leigh Research Ethics Committee. 
Table 1. Demographic and clinical details of all patients.

\begin{tabular}{|c|c|c|c|}
\hline & $\begin{array}{c}\text { All, } \\
\mathrm{n}=170\end{array}$ & $\begin{array}{c}\text { Primary RP, } \\
n=67\end{array}$ & $\begin{array}{c}\text { Secondary RP, } \\
n=103\end{array}$ \\
\hline Median age (range), yrs & $46(18-82)$ & $43(18-78)$ & $51(18-82)$ \\
\hline Sex, male:female & $34: 136$ & $15: 52$ & 19:84 \\
\hline Vasoactive drugs, n (\%) & $57(34)$ & $8(12)$ & $49(48)$ \\
\hline \multicolumn{4}{|l|}{ Underlying disorders } \\
\hline Systemic sclerosis, n (\%) & - & - & $47(47)$ \\
\hline Mixed CTD, n (\%) & - & - & $6(6)$ \\
\hline Undifferentiated CTD, n (\%) & - & - & $39(39)$ \\
\hline Systemic lupus erythematosus, $\mathrm{n}(\%)$ & - & - & $3(3)$ \\
\hline Sjögren's syndrome, n (\%) & - & - & $1(1)$ \\
\hline Polymyositis, $\mathrm{n}(\%)$ & - & - & $1(1)$ \\
\hline Hand arm vibration syndrome, $\mathrm{n}(\%)$ & - & - & $4(4)$ \\
\hline Psoriatic arthritis, $\mathrm{n}(\%)$ & - & - & $1(1)$ \\
\hline Juvenile idiopathic arthritis, n (\%) & - & - & $1(1)$ \\
\hline
\end{tabular}

Thermography protocol. Thermography images were retrieved from the vascular laboratory database at Salford Royal Hospital. Thermography was performed by senior vascular technicians using a standard protocol. An Agema 570 infrared thermography camera (Flir Systems Ltd., West Malling, UK) and the Agema Research 2.1 software were used. Patients were asked to refrain from caffeine/nicotine for $4 \mathrm{~h}$ prior to testing. After acclimatization at $23^{\circ} \mathrm{C}$ for $20 \mathrm{~min}$ in a temperature controlled room an image of the dorsum of each hand was taken, and from these hand images the DDD was calculated, i.e., the temperature of the tip of the fingers minus the temperature of the dorsum of the hand. A DDD of $-1^{\circ} \mathrm{C}$ or less at $23^{\circ} \mathrm{C}$ was prespecified to be considered clinically relevant involvement ${ }^{3,4}$. The worse score (the lower score, i.e., the more negative value) from each pair of digits (right or left hand) was considered for analysis.

Sample size. Assuming there would be double the number of cases with SRP as PRP, it was calculated that 48 patients with PRP and 96 with SRP would allow detection of a difference of $0.5 \mathrm{SD}$ in the outcome (i.e., the average temperature gradient along the thumbs) with $80 \%$ power. We identified 67 patients with PRP and 103 with SRP, giving 88\% power.

Statistical analysis. The results were analyzed using repeated-measures analyses of variance (ANOVA), independent samples $t$ tests, and chi-square tests in SPSS version 16.

\section{RESULTS}

Patients. Demographic details of the 170 patients whose thermography results were included are summarized in Table 1 . The median age of patients was 46 years (range $18-78$ yrs). Sixty-seven (52 women, 15 men) had PRP and 103 (84 women, 19 men) had SRP. Patients with SRP were older than patients with PRP. Forty-seven (41 women, 6 men) had SSc. Thirty-nine patients ( 30 women, 9 men) who had SRP but did not have a specific CTD were classified as having a UCTD. All patients classified as UCTD were ANA-positive. Details of other disorders are summarized in Table 1. Fifty-seven patients (33\%) were receiving vasoactive drugs such as calcium-channel blockers and angiotensin-converting enzyme inhibitors. Of the 57 patients who were taking vasoactive drugs, 8 (14\%) fulfilled the criteria for PRP and 49 (86\%) SRP.

Thermography results. The thumbs were significantly warmer compared to other digits in both PRP and SRP, i.e., the DDD at $23^{\circ} \mathrm{C}$ for the thumbs were significantly higher compared with other digits $(\mathrm{p}<0.001$; Figure 1). Comparison of the DDD at $23^{\circ} \mathrm{C}$ of each digit between PRP and SRP showed all digits were significantly warmer in PRP compared to SRP, with the exception of the thumbs, which were of borderline significance ( $\mathrm{p}=0.07$; Figure 1, Table 2). This could be due to the degree of thumb-sparing being more pronounced in SRP compared with PRP. The proportion of patients with clinically relevant DDD (DDD at $23^{\circ} \mathrm{C}$ of $-1^{\circ} \mathrm{C}$ or less) in thumbs was significantly higher in SRP compared to PRP ( $p=0.003$; Table 3$)$. This difference in the proportion of patients (PRP vs SRP) with a clinically relevant DDD was more pronounced in the thumbs than in other digits (Table 3 ).

\section{DISCUSSION}

This is the first study to show that the degree of thumb involvement differs between PRP and SRP. We found significantly greater involvement of the thumb in SRP compared with PRP as evidenced by a significantly higher proportion of patients who had clinically relevant DDD at $23^{\circ} \mathrm{C}$. A DDD of $-1^{\circ} \mathrm{C}$ or less (dorsum warmer than the tip of fingers) was taken as clinically relevant ${ }^{3,4}$, as in our previous study ${ }^{2}$.

It was not the purpose of our study to distinguish PRP from SRP. However, although not conclusive, thumb involvement should alert rheumatologists to search for an underlying CTD. Other features of RP that should alert a rheumatologist to the possibility of an underlying CTD include disease onset at age $>30$ years, severe episodes, asymmetrical involvement, digital ulcerations/necrosis, abnormal nailfold capillaries, and positive serology such as $\mathrm{ANA}^{5}$. Some features are better predictors for development of CTD than others; for example, abnormal nailfold capillaries in a patient with RP are a better predictor of development of a CTD than a positive ANA ${ }^{6,7}$. The distinction between PRP and SRP is important in view of the prognostic differences between the 2 groups. Patients with PRP tend to follow a mild clinical course, in contrast to the situation Personal non-commercial use only. The Journal of Rheumatology Copyright (C) 2010. All rights reserved. 


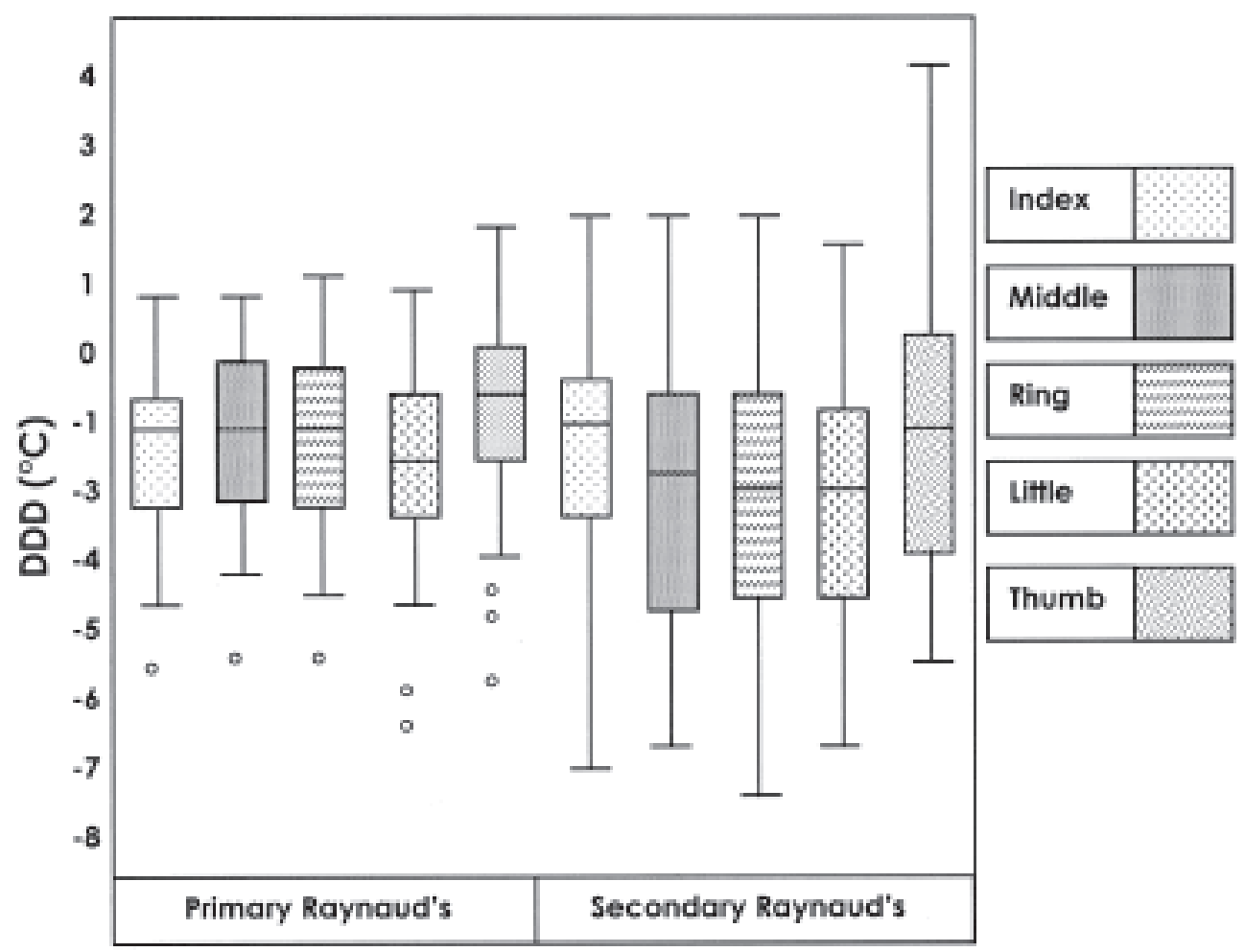

Figure 1. The distribution of worse distal dorsal difference (DDD) scores of each pair of digits, allowing comparison between the thumb and other digits and comparison between primary RP $(n=67)$ and secondary RP $(n=103)$. Upper and lower ends of whiskers represent highest and lowest values, respectively; upper and lower ends of the box represent 75 th and 25th centiles; horizontal line represents the median score; circles represent outliers.

Table 2. Comparison of mean distal dorsal differences at $23^{\circ} \mathrm{C}$ for each digit between primary RP and secondary RP.

\begin{tabular}{lcccc}
\hline & $\begin{array}{c}\text { PRP, } \\
\text { Mean (SD) }\end{array}$ & $\begin{array}{c}\text { SRP, } \\
\text { Mean (SD) }\end{array}$ & $\begin{array}{c}\text { Difference, } \\
\text { Mean (95\% CI) }\end{array}$ & $\mathrm{p}$ \\
\hline Thumb & $-0.87(1.49)$ & $-1.37(1.91)$ & $0.50(-0.04$ to 1.05$)$ & 0.07 \\
Index & $-1.47(1.36)$ & $-2.17(2.02)$ & $0.70(0.15$ to 1.26$)$ & 0.01 \\
Middle & $-1.38(1.35)$ & $-2.40(1.93)$ & $1.01(0.48$ to 1.55$)$ & $<0.001$ \\
Ring & $-1.37(1.45)$ & $-2.31(2.00)$ & $0.94(0.38$ to 1.49$)$ & 0.001 \\
Little & $-1.69(1.43)$ & $-2.39(1.77)$ & $0.70(0.19$ to 1.21$)$ & 0.007 \\
\hline
\end{tabular}

Table 3. Comparison of clinically relevant distal dorsal differences at $23^{\circ} \mathrm{C}$ for each digit between primary RP and secondary RP.

\begin{tabular}{lcccc}
\hline Finger & $\begin{array}{c}\text { Primary, } \\
\mathrm{n}=67\end{array}$ & $\begin{array}{c}\text { Secondary, } \\
\mathrm{n}=103\end{array}$ & OR $(95 \% \mathrm{CI})$ & $\mathrm{p}$ \\
\hline Thumb & $20(30)$ & $55(53)$ & $2.7(1.4$ to 5.2$)$ & 0.003 \\
Index & $39(58)$ & $66(64)$ & $1.3(0.68$ to 2.4$)$ & 0.44 \\
Middle & $37(55)$ & $75(73)$ & $2.2(1.1$ to 4.2$)$ & 0.02 \\
Ring & $36(54)$ & $72(70)$ & $2.0(1.1$ to 3.8$)$ & 0.03 \\
Little & $42(63)$ & $77(75)$ & $1.8(0.91$ to 3.4$)$ & 0.09 \\
\hline
\end{tabular}

in patients with SRP, who may develop severe symptoms accompanied by digital ischemia ${ }^{8}$.

The difference in the prevalence of thumb involvement between PRP and SRP could also point to different patho- physiologies in PRP and SRP. The reasons for these differences were not addressed in this study. Our observations might mean that the thumb is less susceptible to reversible vasospasm that is a characteristic feature of PRP. An obliterative vasculopathy, one of the features of SSc, may be an important contributory factor in thumb involvement in SRP related to CTD.

Symptoms reported by patients could not be included in this retrospective study. However, in our previous prospective study we demonstrated reliability in symptom-reporting using thermography ${ }^{2}$.

Vasoactive drugs can potentially influence thermographic findings; for example, nifedipine has been shown to protect against a reduction in blood flow following a cold challenge in patients with $\mathrm{RP}^{9}$. Several of our patients were receiving vasodilator therapy; thus it is possible that this might have led to underestimation of patients with clinically relevant DDD in temperature, particularly in the SRP group, $48 \%$ of whom were on vasodilator therapy. Whether vasodilator therapy might have preferential effects on different digits is not known, but seems unlikely.

Thumb involvement may be of prognostic significance regarding the possibility of an underlying connective tissue disease or disorder; prospective studies are required to address these questions. 


\section{REFERENCES}

1. LeRoy E, Medsger TJ. Raynaud's phenomenon: a proposal for classification. Clin Exp Rheumatol 1992;10:485-8.

2. Chikura B, Moore TL, Manning JB, Vail A, Herrick AL. Sparing of the thumb in Raynaud's phenomenon. Rheumatology 2008;47:219-21.

3. Anderson ME, Moore TL, Lunt M, Herrick AL. The 'distal-dorsal difference': a thermographic parameter by which to differentiate between primary and secondary Raynaud's phenomenon. Rheumatology 2007;46:533-8.

4. Clark S, Hollis S, Campbell F, Moore T, Jayson M, Herrick A. The "distal-dorsal difference" as a possible predictor of secondary Raynaud's phenomenon. J Rheumatol 1999;26:1125-8.

5. Kallenberg CG. Early detection of connective tissue disease in patients with Raynaud's phenomenon. Rheum Dis Clin North Am 1990;16:11-30.
6. Meli M, Gitzelmann G, Koppensteiner R, Mann-Vesti BR. Predictive value of nailfold capillaroscopy in patients with Raynaud's phenomenon. Clin Rheumatol 2006;25:153-8.

7. Maricq HR, Weinberger AB, Leroy EC. Early detection of scleroderma-spectrum disorders by in vivo capillary microscopy: a prospective study of patients with Raynaud's phenomenon. J Rheumatol 1982;9:289-91.

8. Suter LG, Murabito JM, Felson DT, Fraenkel L. The incidence and natural history of Raynaud's phenomenon in the community. Arthritis Rheum 2005;52:1259-63.

9. Gush RJ, Taylor LJ, Jayson MI. Acute effects of sublingual nifedipine in patients with Raynaud's phenomenon. J Cardiovasc Pharmacol 1987;9:628-31. 